\title{
EDITORIAL
}

\section{EMSec: real time services for maritime safety and security}

\author{
Olga Trivailo $^{1} \cdot$ Stephan Brusch ${ }^{2}$
}

Published online: 19 February 2019

(c) CEAS 2019

EMSec is a BMBF (Federal Ministry of Research and Education) funded project started in 2013, aiming to demonstrate the utility and value of integrating information derived by earth observation data with conventional data streams for improved maritime control and maritime situation awareness. The target is to initiate a coordinated service network to provide all the national users with integrated maritime services in near-real time. The central objective of EMSec is to characterize the added value from combining information derived from satellite and aircraft-based earth observation data with conventional data streams such as AIS (Automatic Identification System), coastal radars, and intelligence sources for maritime situation awareness. By demonstrating the impact and benefits from combining earth observation data derived with conventional system-based information in near-real time due to a human-machine-interface, EMSec is intended to provide clear and credible responses and contributions to the four main areas of criminal activities, natural hazards, maritime casualty and collision, and hazardous materials.

The EMSec Project aims at identifying and developing new tools which provide effective improvements to the stateof-the-art capabilities in maritime surveillance with respect to users' real needs achieved through filling present technological gaps, prominent examples of which include improved secure navigation, protection of navigation systems (spoofing, jamming), improved detection rates of suspicious targets, improved detection quality of hazardous materials and classification, improved anomaly detection and data fusion, improved near-real time capabilities, and development of human-machine-interfaces ${ }^{1}$.

In this Special Issue, nine original papers are presented which provide a comprehensive overview of EMSec relevant techniques and methods, as well as enabling technologies for real-time applications. Some highlighted examples include, amongst others, work on a real-time remote sensing system for maritime security applications, ship detectability depending on TerraSAR-X-derived metocean parameters, automatic bathymetry retrieval from SAR images, and enabling maritime communication and navigation technologies. We would like to sincerely thank all of the authors who contributed their expertise and research to the compilation of this Special Issue on maritime safety and security.

Publisher's Note Springer Nature remains neutral with regard to jurisdictional claims in published maps and institutional affiliations.
Olga Trivailo

olga.trivailo@dlr.de

Stephan Brusch

stephan.brusch@dlr.de

1 Programme Strategy, Executive Board Division for Space Research and Technology, German Aerospace Center, DLR, Hansestr. 115, 51149 Cologne, Germany

2 Programme Coordination for Security Research, Linder Hoehe, 51147 Cologne, Germany

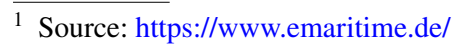

